seroreactivity. Estimates were weighted by the inverse of the participant social network size.

Results Mean age was 29.3 years (range: 18-62), 74.0\% were males, and $89.8 \%$ were non-white. Syphilis seroreactivity was 16.6\%. Being female (wOR: 2.14, 95\% CI: 1.09-4.20), over 29 years old (wOR: 4.44, 95\% CI: 2.41-8.19), exchange sex for money or drugs (wOR: 3.51, 95\% CI: 1.84-6.71), "No/low" self-perceived risk of HIV infection (wOR: 5.13, 95\% CI: 1.3619.37), and having nine or less years of education (wOR: 2.92, 95\% CI: 1.08-7.88) were associated with syphilis seroreactivity. Conclusion One of the most urgent needs for syphilis prevention and control is the availability of rapid point-of-care diagnostic tests and treatment, including the integration of STIs services into primary health-care and HIV-care/prevention settings. Gender issues, education in health, access to public services, social interventions focusing human rights, and social inequalities need more coverage for most at risk populations.

Disclosure of interest statement This study was funded by the Brazilian Ministry of Health/Department of SDT/AIDS and viral hepatitis, with technical advisor from CDC Brazil. The first author is supported by a grant from the Brazilian National Research Council (CNPq) for his PhD studies.

\section{P09.32 CORRELATES OF HIV TESTING AMONG POLYSUBSTANCE USERS IN 10 BRAZILIAN CITIES: A RESPONDENT DRIVEN SAMPLING SURVEY}

${ }^{1} \mathrm{CJ}$ Baptista*, ${ }^{1}$ I Dourado, ${ }^{2} \mathrm{TM}$ Andrade, ${ }^{1} \mathrm{~S}$ Brignol, ${ }^{3} \mathrm{Fl}$ Bastos. 'Instituto de Saúde Coletiva, Universidade Federal Da Bahia; ${ }^{2}$ Faculdade de Medicina Da Bahia, Universidade Federal Da Bahia; ${ }^{3}$ Instituto de Comunicação E Informação Científica E Tecnológica Em Saúde, Fiocruz

\subsection{6/sextrans-2015-052270.416}

Introduction The goal to end HIV/aids pandemic by 2030 has set a number of policies such as $90 \%$ of people living with HIV knowing their HIV status, therefore early diagnosisis is fundamental as component of a comprehensive HIV prevention. In Brazil, 33.6\% of interviewees in the general population reported ever been tested for HIV in 2005. Little is known about HIV testing among illicit polysubstance users (PSUs). We aim to assess correlates of never been tested for HIV among illicit PSUs.

Methods A cross-sectional study recruited 3.449 PSUs in 10 Brazilian cities through respondent driven sampling in 2009. Data was collected using computer-assisted self-interview. To determine correlation, multivariate logistic regression with adjusted odds ratio (aOR, 95\% confidence intervals) was performed. Estimates were weighted by the inverse of the participant social network size.

Results Overall, 56\% had never tested for HIV. Statistically significant odds ratios of never tested were: being male $0.43(0.36-$ $0.51)$, non-white $0.75(0.64-0.88),<=30$ years old 0.65 (0.57-0.76), income <US\$200 (0.71, 0.60-0.86), no-income $0.62(0.51-0.74),<=10$ years of education $(0.82,0.69-0.98)$, and no formal education $(0.48,0.35-0.65)$. Never testing for HIV were also correlated to the following factors: sex exchange $(0.85,0.73-0.98)$, disagree that sex without condom can transmit HIV $(0.30,0.18-0.50)$ and that people apparently healthy should be HIV infected $(0.53,0.36-0.77)$; not receiving counselling on STIs in the last 12 months before the interview $(0.73$, 0.61-0.88), not knowing free HIV testing services $(0.22,0.19-$ $0.26)$, and never had syphilis in lifetime $(0.77,0.60-0.99)$.

Conclusion There is a need to increase access to and uptake of HIV testing among PSUs in Brazil. The availability of self-testing is a possibility to further expand early diagnosis of HIV. However, sociodemographic disparities, stigma and discrimination among PSUs need to be addressed before implementation.

Disclosure of interest statement This study was funded by the Brazilian Ministry of Health/Department of SDT/AIDS and viral hepatitis, with technical advisor from CDC Brazil. The first author is supported by a grant from the Brazilian National Research Council (CNPq) for his PhD studies.

\section{P09.33 SEXUAL BEHAVIOURS AND UNPROTECTED SEX WITH CASUAL AND COMMERCIAL PARTNERS AMONG POLYSUBSTANCE USERS IN BRAZIL: FINDINGS FROM A RESPONDENT DRIVEN SAMPLING STUDY}

${ }^{1} \mathrm{CJ}$ Baptista*, ${ }^{1} \mathrm{I}$ Dourado, ${ }^{2} \mathrm{TM}$ Andrade, ${ }^{1} \mathrm{~S}$ Brignol, ${ }^{3} \mathrm{FI}$ Bastos. ${ }^{1}$ Instituto de Saúde Coletiva, Universidade Federal Da Bahia; ${ }^{2}$ Faculdade de Medicina Da Bahia, Universidade Federal Da Bahia; ${ }^{3}$ Instituto de Comunicação E Informação Científica E Tecnológica Em Saúde, Fiocruz

\subsection{6/sextrans-2015-052270.417}

Introduction Unprotected sexual intercourse (USI) is a known risk factor for HIV infection. And bisexual men (BSM) who engage in USI with casual and commercial partners may be at a higher risk. Our aim is to estimate prevalence of men having sex with men (MSM), BSM, and USI to guide prevention via sexual route among polysubstance users (PSUs).

Methods A total of 3.449 PSUs were recruited in a cross-sectional study in 10 Brazilian cities through respondent driven sampling in 2009. Data was collected using audio computerassisted self-interview. Estimates were weighted by the inverse of the participant's social network size.

Results Among 2,682 (73.7\% of the sample) males, $4.4 \%$ referred having sex only with men (MSM), 16.2\% with both men and women (BSM). 64.8\% engaged in USI with commercial partnerships; whereas $47.1 \%$ of those exchanged sex for money/ drugs 12 months before the interview. Casual partnerships 12 months before the interview were reported by $47.9 \%$ of men; whereas $62.3 \%$ of those reported USI with that casual partners. Conclusion It is urgent to design interventions among males PSUs in Brazil who engage in commercial and casual USI. Social interventions, education in health, and linkage to health services are needed to prevent sexual transmission of HIV in this group most at risk.

Disclosure of interest statement This study was funded by the Brazilian Ministry of Health/Department of SDT/AIDS and viral hepatitis, with technical advisor from CDC Brazil. The first author is supported by a grant from the Brazilian National Research Council (CNPq) for his PhD studies.

\section{P09.34 MOLECULAR IDENTIFICATION OF TREPONEMA PALLIDUM STRAINS FROM SYPHILITIC LESIONS IN STI CLINIC PATIENTS, LIMA, PERU}

${ }^{1} J A$ Flores ${ }^{*},{ }^{1}$ SK Vargas, ${ }^{1}$ SR Leon, ${ }^{1,2}$ KA Konda, ${ }^{2} \mathrm{JY}$ Chow, ${ }^{3} \mathrm{GM}$ Calvo, ${ }^{4} \mathrm{HJ}$ Salvatierra, ${ }^{5} \mathrm{~B}$ Brown, ${ }^{2} \mathrm{JD}$ Klausner, 'CF Caceres. 'Unit of Health, Sexuality and Human Development, and Laboratory of Sexual Health, Universidad Peruana Cayetano Heredia, Lima, Peru; 'Division of Infectious Diseases, David Geffen School of Medicine, University of California, Los Angeles, CA, USA; ${ }^{3}$ Epicentro, Lima, Peru; ${ }^{4}$ Alberto Barton Health Center, Health Directorate of Callao, Lima, Peru; ${ }^{5}$ School of Public Health, University of California, IIvine, CA, USA

10.1136/sextrans-2015-052270.418 
Background In Peru, syphilis disproportionally affects men who have sex with men (MSM) and male-to-female transgender women (TW) with prevalence rates as high as $21 \%$. To our knowledge, there are no available data describing circulating strains of T. pallidum in Lima, Peru. We used the CDC subtyping scheme to identify T. pallidum circulating subtypes among MSM and TW from two STI clinics in Lima, Peru.

Methods A cohort of 401 MSM and TW were assessed for syphilis infection at baseline and quarterly with RPR (BD Macro-Vue, USA) and TPPA (Fujirebio, Japan) testing up to 24 months. A dacron swab was used to collect exudate from chancre-like lesions and placed into $500 \mu \mathrm{L}$ of lysis buffer. DNA extraction was performed using QIAamp mini kit (Qiagen, Valencia, CA). Using specific primers for $\mathrm{Tp} 47$ region target, an aliquot of the DNA sample was amplified using conventional PCR. Subtyping of T. pallidum TP47 positives was performed using detection of number of 60 -bp tandem repeats in the arp gene and analysis by RFLP of 3 tpr genes (TprE, G, J) according to CDC guidelines.

Results Among 401 participants, 26 presented with primary syphilitic lesions at baseline or follow-up with RPR (TPPA confirmed) titers ranging from 1:2-1:64. Of those 26 total lesions, $1(7 \%)$ of 14 tested was dark-field positive. TP DNA screening using TP47 PCR yielded 12 (44.4\%) positives. Among eight typable, four were subypes $14 \mathrm{~d}(33.3 \%)$, two $15 \mathrm{~d}(16.7 \%)$, one $16 \mathrm{~d}(8.3 \%)$ and one $0 \mathrm{~d}(16.7 \%)$ where $0=$ non-typable arp.

Conclusion T. pallidum subtypes $14 \mathrm{~d}$ and $15 \mathrm{~d}$ were the most prevalent strains in lesions obtained from MSM/TW who had chancre-like lesions in Lima, Peru. Dark field testing results and typing difficulties could be due to low bacterial load and needs to be considered in sampling methods.

Disclosure of interest statement The study Picasso is funded by a NIAID grant and was implemented by Cayetano Heredia in collaboration with the University of California Los Ángeles. The molecular part of the study was implemented under the supervision of the University of Washington. No pharmaceutical grants were received during the development of this study.

\section{P09.35 ERADICATION OF SYPHILIS? - NOT THERE YET ONGOING PUBLIC HEALTH RESPONSE TO A SYPHILIS OUTBREAK IN THE NORTHERN TERRITORY, AUSTRALIA}

${ }^{1} \mathrm{~L}$ Garton*, ${ }^{1} \mathrm{M}$ Gunathilake, ${ }^{1} \mathrm{JY}$ Su, ${ }^{1} \mathrm{M}$ Russell, ${ }^{1} \mathrm{TW}$ Yip, ${ }^{2} \mathrm{~A}$ Hope, ${ }^{3} \mathrm{~N}$ Ryder, ${ }^{1} \mathrm{M}$ Thalanany, ${ }^{1} \mathrm{~V}$ Krause. ${ }^{1}$ Centre of Disease Control, Darwin, Northern Territory; ${ }^{2}$ Aboriginal Medical Services Alliance, Northern Territory; ${ }^{3}$ Newcastle Sexual Health Services, Hunter New England Local Health District, NSW

10.1136/sextrans-2015-052270.419

Introduction An extensive outbreak of infectious syphilis in the Northern Territory (NT) was identified in Central Australia and Katherine regions in 2014. The NT Centre for Disease Control (CDC) launched an outbreak response to contain it.

Methods A multi-disciplinary outbreak response team was formed to drive the response. Comprehensive testing and treatment data were regularly collected and used to monitor the outbreak and to guide the response measures. Opportunistic testing was proactively promoted in affected regions. In areas with high numbers of cases and untraceable contacts, a community-wide screen using a point-of-care test (PoCT) for syphilis was conducted for those aged 12-30 years. Local guidelines were followed in treatment and contact tracing. Updates on the outbreak response were communicated to the affected communities regularly.
Results As of 7 April 2015, 112 cases (60 females and 52 males) of infectious syphilis were detected, consisting of 74 and 38 confirmed and probable cases, respectively. Their ages ranged from 12 to 37 years (median: 17; inter-quartile range: 15-20.5). Community-wide screening was conducted in 2 remote Aboriginal communities with combined population coverage of $62.7 \%$ and a prevalence of $8.4 \%$ in the targeted age group (12-30 years). All positive PoCT results were confirmed by normal syphilis serology tests.

Conclusion Effective syphilis outbreak control requires up-todate surveillance on testing and treatment as well as an outbreak response team capable of adopting the best disease control measures in a timely fashion to the different stages and needs of the outbreak. In particular, using PoCT for community screening is proving to be an extremely useful case finding method, shortening the time to treatment and thus the period of infectiousness in communities most affected. Further control of the outbreak will require inter-jurisdictional collaboration.

Disclosure of interest statement There are no conflicts of interest

\section{P09.36 IMPROVING UPTAKE, INTERPRETATION AND QUALITY OF SYPHILIS TESTING IN THE AMERICAS THROUGH THE DEVELOPMENT OF A NEW REGIONAL GUIDANCE DOCUMENT}

${ }^{1}$ Freddy Perez* ${ }^{2}$ Adele Schwartz Benzaken, ${ }^{3}$ Kevin Karem, 'Jorge Matheu, ${ }^{3}$ Mary Kamb. 'HIV, Hepatitis, Tuberculosis and Sexually Transmitted Infectious Unit-Communicable Diseases and Health Analysis Department- Pan American Health Organization; 'Department of STI, AIDS and Viral Hepatitis, Health Surveillance Unit, Ministry of Health of Brazil; ${ }^{3}$ Centers for Disease Control and Prevention (CDC), Division of Sexually Transmitted Disease Prevention, National Center for HIVIAIDS, Viral Hepatitis, STD and TB Prevention

\subsection{6/sextrans-2015-052270.420}

Introduction In 2009, an initiative to eliminate mother-to-child transmission (MTCT) of HIV and congenital syphilis was launched in the Americas. The region sought to develop means of increasing uptake of syphilis testing for pregnant women and key populations at risk for syphilis to ensure prompt detection and treatment with minimal loss to follow-up.

Methods For a regional consultation, a PAHO/CDC team reviewed the published and grey literature on syphilis testing to identify regional standards and gaps. In April 2014, a regional meeting was held with representatives from health ministries and technical experts in maternal and child health and laboratory diagnostics. Participants reviewed and discussed the data, reported on best practices, and identified key areas to be included in a regional policy document.

Results Participants raised several concerns, such as a lack of regional or global guidance on syphilis testing strategies for specific clinical settings being a barrier impeding elimination of MTCT of HIV and syphilis. Also, limited integration of HIV and syphilis program and operations' systems was a "missed opportunity" to leverage efficiencies, personnel and funding in many countries. Furthermore, existing "best practices" promoting syphilis testing in different clinical settings had not been widely circulated. Participants recommended the development of a regional consensus document. In March 2015, this Guidance outlining syphilis testing algorithms in different clinical settings was published emphasising 5 areas for national programs: (1) comprehensive national policies on syphilis testing and treatment; (2) syphilis testing algorithms appropriate for specific populations or clinical (or outreach) settings, depending on laboratory capacity (e.g., rapid syphilis testing strategies); (3) 